\title{
Efficient cyclic reduction for Quasi-Birth-Death problems with rank structured blocks
}

\author{
Dario A. Bini ${ }^{\mathrm{a}}$, Stefano Massei ${ }^{\mathrm{b}, *}$, Leonardo Robol $^{\mathrm{c}}$ \\ a Dipartimento di Matematica, University of Pisa, Italy \\ b Scuola Normale Superiore, Pisa, Italy \\ c Department of Computer Science, KU Leuven, Belgium
}

\section{A R T I C L E I N F O}

\section{Article history:}

Available online $\mathrm{xxxx}$

\section{Keywords:}

Cyclic reduction

Matrix equations

Double QBD processes

Markov chains

$\mathrm{H}$ matrices

Quasiseparable matrices

\begin{abstract}
A B S T R A C T
We provide effective algorithms for solving block tridiagonal block Toeplitz systems with $m \times m$ quasiseparable blocks, as well as quadratic matrix equations with $m \times m$ quasiseparable coefficients, based on cyclic reduction and on the technology of rankstructured matrices. The algorithms rely on the exponential decay of the singular values of the off-diagonal submatrices generated by cyclic reduction. We provide a formal proof of this decay in the Markovian framework. The results of the numerical experiments that we report confirm a significant speed up over the general algorithms, already starting with the moderately small size $m \approx 10^{2}$.
\end{abstract}

(C) 2016 IMACS. Published by Elsevier B.V. All rights reserved.

\section{Introduction}

Cyclic reduction (CR) is an effective tool that can be used for solving several problems in linear algebra and in polynomial computations [4]. It has been originally introduced by G.H. Golub and R.W. Hockney in the mid 1960s [14,8], for the numerical solution of block tridiagonal linear systems stemming from the finite differences solution of elliptic problems, and has been generalized to solve nonlinear matrix equations associated with matrix power series with applications to queuing problems, Markov chains and spectral decomposition of polynomials. In fact, an important application of $\mathrm{CR}$ concerns the computation of the minimal nonnegative solution of the matrix equation $X=A_{-1}+A_{0} X+A_{1} X^{2}$, encountered in Quasi Birth-Death (QBD) Markov chains, where $A_{-1}, A_{0}$, and $A_{1}$ are given $m \times m$ nonnegative matrices such that $A_{-1}+A_{0}+A_{1}$ is irreducible and stochastic and where $X$ is the matrix unknown [2,4]. The computation of the solution $X$ allows to recover the steady state vector $\pi$ of the Markov chain.

Rewriting the matrix equation as $A_{-1}+\left(A_{0}-I\right) X+A_{1} X^{2}=0$, CR computes four sequences of matrices, $A_{i}^{(h)}, i=-1,0,1$ and $\widehat{A}_{0}^{(h)}$, according to the following equations

$$
\begin{aligned}
& A_{1}^{(h+1)}=-A_{1}^{(h)} S^{(h)} A_{1}^{(h)}, \quad S^{(h)}=\left(A_{0}^{(h)}-I\right)^{-1} \\
& A_{0}^{(h+1)}=A_{0}^{(h)}-A_{1}^{(h)} S^{(h)} A_{-1}^{(h)}-A_{-1}^{(h)} S^{(h)} A_{1}^{(h)}, \\
& A_{-1}^{(h+1)}=-A_{-1}^{(h)} S^{(h)} A_{-1}^{(h)}, \quad \widehat{A}_{0}^{(h+1)}=\widehat{A}_{0}^{(h)}-A_{1}^{(h)} S^{(h)} A_{-1}^{(h)},
\end{aligned}
$$

\footnotetext{
Work supported by Gruppo Nazionale di Calcolo Scientifico (GNCS) of INdAM, and by the PRA project "Mathematical models and computational methods for complex networks" funded by the University of Pisa.

* Corresponding author.

E-mail addresses: bini@dm.unipi.it (D.A. Bini), stefano.massei@sns.it (S. Massei), leonardo.robol@sns.it (L. Robol).
} 


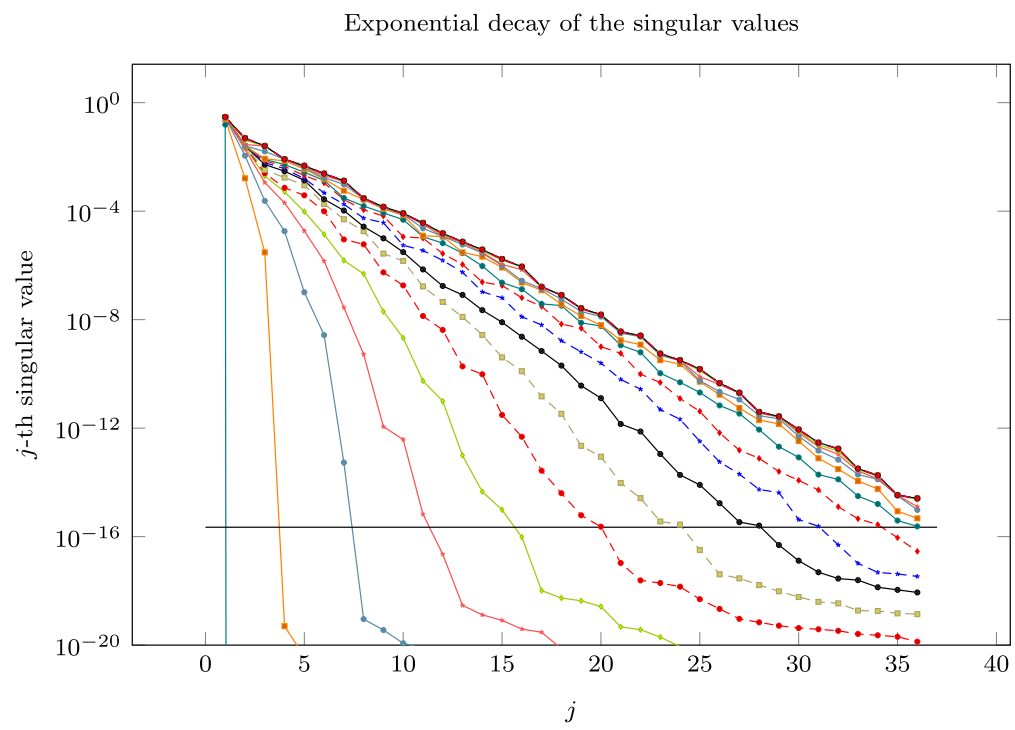

Fig. 1. Log-scale plot of the largest singular values of the largest south-western submatrix of $A_{0}$ contained in the lower triangular part, for $m=1600$. The horizontal line denotes the machine precision threshold. Matrices are randomly generated so that $A_{i} \geqslant 0$ are tridiagonal matrices and $A_{-1}+A_{0}+A_{1}$ is stochastic. (For interpretation of the references to color in this figure, the reader is referred to the web version of this article.)

for $h=0,1, \ldots$, with $A_{i}^{(0)}=A_{i}, i=-1,0,1$ and $\widehat{A}_{0}^{(0)}=A_{0}-I$. It can be proved, in the context of Markov chains, [2] that the sequence $-\left(\widehat{A}_{0}^{(h)}\right)^{-1} A_{-1}$ converges to the minimal nonnegative solution $G$ of the matrix equation.

If the $\mathrm{QBD}$ process is not null recurrent, applicability and quadratic convergence of the algorithm are guaranteed $[2$, Theorems 7.5, 7.6]. In the null recurrent case, it has been proved in [12] that convergence is linear with factor $\frac{1}{2}$.

Without any further assumption on the structure of the blocks, each step of $\mathrm{CR}$ requires a small number of matrix multiplications and one matrix inversion for the resulting computational cost of $O\left(\mathrm{~m}^{3}\right)$ arithmetic operations (ops) per step. On the other hand, there are several models from the applications in which the blocks $A_{i}$ exhibit special structures. In order to decrease the computational complexity of the iterations, variations of CR which exploit these structures have been proposed, see for instance $[3,18,1]$.

Here, we are interested in analyzing the case where the blocks $A_{i}$ are quasiseparable matrices. That is, the case where the off-diagonal submatrices of $A_{-1}, A_{0}$ and $A_{1}$, strictly contained in the upper or in the lower triangular part, have low rank with respect to $m$. The maximum of the ranks of the off-diagonal submatrices is called quasiseparable rank and a matrix with quasiseparable rank $k$ is called $k$-quasiseparable. Observe that $k$-quasiseparable matrices include banded matrices. These structures are encountered in wide and important classes of applications like, bidimensional random walks [17], the Jackson tandem queue model [15] and other QBD processes, or, for instance, in the finite differences discretization of elliptic PDEs.

Our goal is to design a version of $\mathrm{CR}$ which exploits the rank structures of the blocks $A_{i}$ and which can be implemented at a substantially lower cost. This way, we may arrive at designing effective solvers both for block tridiagonal block Toeplitz systems and for the quadratic matrix equations encountered in QBD Markov chains. Indeed, a way to reach this goal is to find out if some structure of the blocks $A_{i}^{(h)}$ is maintained during the CR steps (1), and then to try to exploit this structure in order to design an effective implementation of CR.

Looking at the iterative scheme (1), one can find out that the quasiseparable rank can grow exponentially at each step. Despite that, plotting the singular values of the off-diagonal blocks of the matrices $A_{i}^{(h)}$ shows an interesting behavior as reported in Fig. 1. For a randomly generated QBD process with tridiagonal blocks of size 1600, the singular values of an off-diagonal submatrix of size $799 \times 800$ in $A_{0}^{(h)}$ have an exponential decay in the first 20 steps of CR.

It is evident that, even though the number of nonzero singular values grows at each step of CR, the number of singular values above the machine precision - denoted by a horizontal line in Fig. 1 - is bounded by a moderate constant. Moreover, the singular values seem to stay below a straight-line which constitutes an asymptotic bound. That is, they get closer to this line as $h \rightarrow \infty$. The logarithm scale suggest that the computed singular values $\sigma_{i}^{(h)}$ decay exponentially with $i$ and the basis of the exponential grows with $h$ but has a limit less than 1 .

We will prove this asymptotic property relying on the technology of rank-structured matrices and relate the basis of the exponential decay to the width of the domain of analyticity of the matrix function $\varphi(z)^{-1}$ for $\varphi(z)=-z^{-1} A_{-1}+I-$ $A_{0}-z A_{1}$.

It is interesting to observe that the three matrix sequences $A_{i}^{(h)}, i=-1,0,1$ are related to certain Schur complements of the block tridiagonal block Toeplitz matrix $\operatorname{trid}\left(A_{-1}, A_{0}, A_{1}\right)$, obtained after permuting block rows and columns with the odd-even permutation [4]. A slightly different sequence is given by the Schur complements obtained by applying Gaussian 
elimination to $\operatorname{trid}\left(A_{-1}, A_{0}, A_{1}\right)$, namely, $V_{h+1}=A_{0}-A_{-1} V_{h}^{-1} A_{1}, V_{0}=A_{0}$. This sequence has been analyzed in [9] where it is shown that, if the blocks $A_{-1}, A_{0}, A_{1}$ come from the finite difference discretization of constant coefficients elliptic PDEs, then the numerical rank of the off diagonal blocks of $V_{h}$ are bounded by a constant independent of the size. This result requires that the blocks $A_{-1}, A_{0}, A_{1}$ be tridiagonal and commute, while our result holds under much weaker conditions and concerns similar Schur complements.

The paper is organized as follows. In Section 2 we recall some useful properties of CR. In Section 3 we prove some preliminary lemmas for bounding the singular values of sums, products and inverses of matrices. Section 4 contains the proof of the main result concerning the exponential decay of the singular values. In Section 5 we describe our algorithm which implements CR relying on the package H2Lib [5], concerning $\mathcal{H}$-matrices, and the related code together with the results of the numerical experiments. Finally, in Section 6, we give some concluding comments and possible future developments.

\section{Main properties of cyclic reduction}

We recall a functional interpretation of cyclic reduction introduced in the Markov chains framework [2,4] to prove applicability and convergence properties.

Associate the matrices $A_{i}^{(h)}, i=-1,0,1$ defined in (1) with the matrix Laurent polynomial $\varphi^{(h)}(z):=-z^{-1} A_{-1}^{(h)}+(I-$ $\left.A_{0}^{(h)}\right)-z A_{1}^{(h)}$, where $\varphi^{(0)}(z)=\varphi(z)=z^{-1} A_{-1}+\left(I-A_{0}\right)-z A_{1}$, and define the matrix rational function $\psi^{(h)}(z)=\varphi^{(h)}(z)^{-1}$. The following property holds

$$
\left\{\begin{array}{l}
\psi^{(0)}(z):=\psi(z) \\
\psi^{(h+1)}\left(z^{2}\right):=\frac{1}{2}\left(\psi^{(h)}(z)+\psi^{(h)}(-z)\right)
\end{array}\right.
$$

In particular, making explicit the recurrence relation in the sequence $\left\{\psi^{(h)}\right\}$, we find that $\psi^{(h)}\left(z^{2^{h}}\right)=\frac{1}{2^{h}} \sum_{j=0}^{2^{h}-1} \psi^{(0)}\left(\omega_{N}^{j} z\right)$ where $\omega_{N}=e^{\frac{2 \pi}{N} \boldsymbol{i}}$ is a principal $N$-th root of the unity for $N=2^{h}$, and $\boldsymbol{i}$ denotes the imaginary unit, so that

$$
\varphi^{(h)}\left(z^{2^{h}}\right)=\left(\frac{1}{2^{h}} \sum_{j=0}^{2^{h}-1} \psi^{(0)}\left(\omega_{N}^{j} z\right)\right)^{-1}
$$

Observe that in the case where $A_{-1}, A_{0}$ and $A_{1}$ are tridiagonal, then $\varphi(z)$ is tridiagonal as well, so that for any value of $z$ such that $\operatorname{det} \varphi(z) \neq 0$, the matrix $\psi(z)$ is quasiseparable, that is, $\operatorname{tril}(\psi(z))=\operatorname{tril}(L)$, $\operatorname{triu}(\psi(z))=\operatorname{triu}(U)$, where $L$ and $U$ are matrices of rank 1 [19].

Relying on this property, in the next section we will show that $\psi_{h}(z)$, as well as the blocks $A_{-1}^{(h)}$, $A_{0}^{(h)}$ and $A_{1}^{(h)}$, has off-diagonal submatrices with singular values which decay exponentially.

\section{Singular values of sums, products and inverses}

In this section, we recall some basic facts on the singular values decomposition (SVD) and provide some technical lemmas. Let us denote by $\sigma_{j}(A)$ the $j$-th singular value of the $m \times n$ matrix $A$ sorted in non-ascending order and by $A=U \Sigma V^{*}$, $\Sigma=\operatorname{diag}\left(\sigma_{1}, \ldots, \sigma_{p}\right), p=\min \{m, n\}$, the SVD of $A$ where $U$ and $V$ are unitary and $V^{*}$ denotes the transpose conjugate of $V$. Moreover, denote by $u_{i}$ and $v_{i}$ the $i$ th column of $U$ and $V$, respectively. We recall the following well-known property concerning the SVD of a matrix $A$.

Property 3.1. For any matrix $A$ the function $f(X)=\|A-X\|_{2}$ takes its minimum over the class of matrices of rank $\ell-1$ for $X=$ $\sum_{i=1}^{\ell-1} \sigma_{i} u_{i} v_{i}^{*}$ and the value of the minimum is exactly $\sigma_{\ell}$.

\subsection{Some technical lemmas}

Let $\|A\|_{2}$ be the Euclidean norm of $A$ and $\mu_{2}(A)=\|A\|_{2} \cdot\left\|A^{-1}\right\|_{2}$ be the condition number of $A$. The following result relates the singular values of the matrices $A, B$ and $C=A B$.

Lemma 3.2. Consider two matrices $A \in \mathbb{C}^{m \times n}, B \in \mathbb{C}^{n \times n}$, such that $B$ is invertible. Then

$$
\frac{\sigma_{j}(A)}{\left\|B^{-1}\right\|_{2}} \leqslant \sigma_{j}(A B) \leqslant\|B\|_{2} \cdot \sigma_{j}(A), \quad \frac{\sigma_{j}(A)}{\left\|B^{-1}\right\|_{2}} \leqslant \sigma_{j}\left(B A^{*}\right) \leqslant\|B\|_{2} \cdot \sigma_{j}(A),
$$

Proof. The lemma immediately follows by applying the minimax theorem to the matrix $M=(A B)^{*}(A B)$ since the singular values of $A B$ are the square roots of the eigenvalues of $M$.

The following lemma relates the singular values of an off-diagonal submatrix of the inverse of a given matrix $A$ with those of the corresponding submatrix of $A$. 
Lemma 3.3. Let $\mathcal{A} \in \mathbb{C}^{n \times n}$ be an invertible matrix and consider the block partitioning

$$
\mathcal{A}=\left(\begin{array}{cc}
A & B \\
C & D
\end{array}\right), \mathcal{A}^{-1}=\left(\begin{array}{cc}
\tilde{A} & \tilde{B} \\
\tilde{C} & \tilde{D}
\end{array}\right),
$$

where $A$ and $\tilde{A}$ are $i \times i$ matrices for $2 \leqslant i \leqslant n-1$. We have the following properties

1. If $D$ is invertible then $\frac{1}{\|D\|_{2}\left\|S_{D}\right\|_{2}} \sigma_{j}(C) \leqslant \sigma_{j}(\tilde{C}) \leqslant\left\|D^{-1}\right\|_{2} \cdot\left\|S_{D}^{-1}\right\|_{2} \cdot \sigma_{j}(C)$, where $S_{D}=A-B D^{-1} C$ is the Schur complement of $D$.

2. If $A$ is invertible then $\frac{1}{\|A\|_{2}\left\|S_{A}\right\|_{2}} \sigma_{j}(C) \leqslant \sigma_{j}(\tilde{C}) \leqslant\left\|A^{-1}\right\|_{2} \cdot\left\|S_{A}^{-1}\right\|_{2} \cdot \sigma_{j}(C)$, where $S_{A}=D-C A^{-1} B$ is the Schur complement of $A$.

Proof. Let us consider part 1 . If $D$ is invertible we can write

$$
\left(\begin{array}{cc}
A & B \\
C & D
\end{array}\right)^{-1}=\left(\begin{array}{cc}
S_{D}^{-1} & S_{D}^{-1} B D^{-1} \\
-D^{-1} C S_{D}^{-1} & D^{-1}+D^{-1} C S_{D}^{-1} B D^{-1}
\end{array}\right)
$$

and in particular we have $\tilde{C}=-D^{-1} C S_{D}^{-1}$. Repeatedly applying Lemma 3.2 to $\tilde{C}$ yields $\frac{1}{\|D\|_{2}} \sigma_{j}\left(C S_{D}^{-1}\right) \leqslant \sigma_{j}(\tilde{C}) \leqslant\left\|D^{-1}\right\|_{2}$. $\sigma_{j}\left(C S_{D}^{-1}\right)$ and eventually $\frac{1}{\left\|S_{D}\right\|_{2}} \sigma_{j}(C) \leqslant \sigma_{j}\left(C S_{D}^{-1}\right) \leqslant\left\|S_{D}^{-1}\right\|_{2} \cdot \sigma_{j}(C)$. Combining these inequalities gives us the thesis. For proving part 2 , we can proceed in the same manner relying on the inversion formula

$$
\left(\begin{array}{cc}
A & B \\
C & D
\end{array}\right)^{-1}=\left(\begin{array}{cc}
A^{-1}+A^{-1} B S_{A}^{-1} C A^{-1} & -A^{-1} B S_{A}^{-1} \\
-S_{A}^{-1} C A^{-1} & S_{A}^{-1}
\end{array}\right) .
$$

Lemma 3.4. Let $A=\sum_{i=-\infty}^{+\infty} A_{i}$ and $A^{+}=\sum_{i=0}^{+\infty} A_{i}$ where $A_{i} \in \mathbb{C}^{m \times n}$ have rank at most $k$ and suppose that $\left\|A_{i}\right\|_{2} \leqslant M e^{-\alpha|i|}$. Then $\sigma_{j}(A) \leqslant \frac{2 M}{1-e^{-\alpha}} \cdot e^{-\alpha \frac{j-k}{2 k}}, \sigma_{j}\left(A^{+}\right) \leqslant \frac{M}{1-e^{-\alpha}} \cdot e^{-\alpha \frac{j-k}{k}}$.

Proof. Note that $B_{s}=\sum_{i=-s+1}^{s-1} A_{i}$ has rank at most $k(2 s-1)$. For $j$ positive integer, set $s=\left\lceil\frac{j-k}{2 k}\right\rceil$ and observe that, since $2 k\left\lceil\frac{j-k}{2 k}\right\rceil<j+k$, we have $k(2 s-1) \leqslant j-1$ so that $B_{s}$ is an approximation to $A$ of rank at most $j-1$. By Property 3.1 it follows that $\sigma_{j}(A) \leqslant\left\|A-B_{s}\right\|_{2}$. Moreover, since $A-B_{s}=\sum_{|i| \geqslant s} A_{i}$, we have $\sigma_{j}(A) \leqslant \sum_{|i| \geqslant s}\left\|A_{i}\right\|_{2} \leqslant 2 M e^{-\alpha s} /\left(1-e^{-\alpha}\right)$ which completes the proof of the first bound. The second bound is proved similarly.

Remark 3.5. In the particular case where $k=1$, Lemma 3.4 yields $\sigma_{S}(A) \leqslant \frac{2 M}{1-e^{-\alpha}} \cdot e^{-\alpha \frac{s}{2}}, \sigma_{s}\left(A^{+}\right) \leqslant \frac{M}{1-e^{-\alpha}} \cdot e^{-\alpha(s-1)}$.

\section{Exponential decay of the singular values}

We can now state the main result about the decay of the singular values. It is clear that, if the blocks $A_{i} i=-1,0,1$ have an off-diagonal rank structure, then the matrix $\varphi^{(0)}(z)$ also enjoys this property. We will show that this fact implies the exponential decay of the singular values of the off-diagonal blocks of $\varphi^{(h)}\left(z^{2^{h}}\right)$ for every $h$ and for any $z \in \mathbb{T}$ where $\mathbb{T}=\{z \in \mathbb{C}:|z|=1\}$ denotes the unit circle in the complex plane.

Given an integer $N>0$, let $\omega_{N}=e^{i 2 \pi / N}$ and observe that the quantity $\frac{1}{N} \sum_{j=0}^{N-1}\left(z \omega_{N}^{j}\right)^{k}$ coincides with $z^{k}$ if $k \equiv 0$ mod $N$ and with 0 otherwise. This way, if $A(z)=\sum_{i \in \mathbb{Z}} z^{i} A_{i}$ is a matrix Laurent series analytic on the annulus $\mathbb{A}\left(r_{1}, r_{2}\right)=\{z \in$ $\left.\mathbb{C}: r_{1}<|z|<r_{2}\right\}$ for $0<r_{1}<1<r_{2}$, then $\frac{1}{N} \sum_{j=0}^{N-1} A\left(\omega_{n}^{j} z\right)=B\left(z^{N}\right)$ where $B(z)=\sum_{i \in \mathbb{Z}} z^{i} A_{N i}$ is analytic on $\mathbb{A}\left(r_{1}^{N}, r_{2}^{N}\right)$. We denote by $I_{N}$ the operator which maps $A(z)$ into $B(z)$ and write $B(z)=I_{N}(A(z))$. Observe that $I_{N}$ is linear and continuous on the space of analytic functions on $\mathbb{A}\left(r_{1}, r_{2}\right)$.

Moreover, in view of $(2)$, we have $\psi^{(h)}=I_{N}\left(\psi^{(0)}\right)$ for $N=2^{h}$. This way, if we prove that any off-diagonal submatrix $B(z)$ of $\psi^{(0)}(z)$ is such that $I_{N}(B(z))$ has the exponential decay property for its singular values, then we have shown this property also for $\psi^{(h)}(z)$.

Partition $\varphi(z)$ and $\varphi(z)^{-1}$ as follows

$$
\varphi(z)=\left[\begin{array}{cc}
I-E(z) & -B(z) \\
-C(z) & I-D(z)
\end{array}\right], \quad \psi(z):=\varphi(z)^{-1}=\left[\begin{array}{cc}
\tilde{E}(z) & \tilde{B}(z) \\
\tilde{C}(z) & \tilde{D}(z)
\end{array}\right],
$$

where $E(z)$ and $D(z)$ are square matrices of any compatible size.

Theorem 4.1. Let $\varphi(z)=-z^{-1} A_{-1}+I-A_{0}-z A_{1}$ be an $m \times m$ matrix function such that

(i) The $A_{i}$ are non-negative and $I-\varphi(z)$ has spectral radius smaller than 1 for any $z \in \mathbb{T}$.

(ii) The blocks $A_{i}$ are $k$-quasiseparable and $\left\|A_{i}\right\|_{2} \leqslant L, i=-1,0,1$.

(iii) There exist $t>1$ and $\delta>0$ such that $\operatorname{det} \varphi(z) \neq 0$ and $\left\|\varphi(z)^{-1}\right\|_{2} \leqslant \delta$ for $z \in \mathbb{A}\left(t^{-1}, t\right)$. 
Then $\rho(I-\varphi(z))<1$ for any $z \in \mathbb{A}$ and in the partitioning (3), both blocks $I-E(z)$ and $I-D(z)$ are invertible for any $z \in \mathbb{A}$. Moreover, for any $z \in \mathbb{T}$ and for any $h$, the singular values of $\tilde{C}^{(h)}:=I_{N}(\tilde{C}(z))$, with $N=2^{h}$, are such that

$$
\sigma_{s}\left(\tilde{C}^{(h)}(z)\right) \leqslant 3 M e^{-\frac{s-3 k}{6 k} \log t}, \quad M=\frac{4 L \delta^{2}}{\left(1-e^{-N \log t}\right)\left(1-t^{-1}\right)} .
$$

Moreover, if $A_{-1}, A_{0}, A_{1}$ are tridiagonal then the above bound turns into

$$
\sigma_{s}\left(\tilde{C}^{(h)}(z)\right) \leqslant M e^{-\frac{s}{2} \log t} .
$$

Proof. Let us prove that $\rho(I-\varphi(z))<1$ for any $z \in \mathbb{A}$. By contradiction, assume that there exists $\xi \in \mathbb{A}$ such that $\rho(I-$ $\varphi(\xi)) \geqslant 1$. Since $A_{i} \geqslant 0$ for $i=-1,0,1$ then $|I-\varphi(\xi)| \leqslant I-\varphi(|\xi|)$, and by the monotonicity of the spectral radius we get $1 \leqslant \rho(I-\varphi(\xi)) \leqslant \rho(I-\varphi(|\xi|))$. Thus, since $\rho(I-\varphi(1))<1 \leqslant \rho(I-\varphi(|\xi|))$ and $\rho$ is a continuous function, then there exists $1 / t<\hat{\xi}<t$ such that $\rho(I-\varphi(\hat{\xi}))=1$. Since $I-\varphi(\hat{\xi})$ is nonnegative, then by the Perron-Frobenius theorem there exists an eigenvalue of $I-\varphi(\hat{\xi})$ equal to 1 , that is $\varphi(\hat{\xi})$ would be singular, which contradicts the assumptions.

Now we prove that $I-D(z)$ and $I-E(z)$ are invertible for any $z \in \mathbb{A}$. Since $|D(z)| \leqslant D(|z|)$, for the monotonicity of the spectral radius, we have $\rho(D(z)) \leqslant \rho(|D(z)|) \leqslant \rho(D(|z|))$. On the other hand, $D(|z|)$ is a principal submatrix of the nonnegative matrix $I-\varphi(|z|)$ so that $\rho(D(|z|)) \leqslant \rho(I-\varphi(|z|))$ which is less than 1 since $|z| \in \mathbb{A}$. We conclude that $\rho(D(z))<1$ for any $z \in \mathbb{A}$ so that $I-D(z)$ is nonsingular. The same argument can be used to deduce that $I-E(z)$ is nonsingular.

Now we prove the bound (4) on the singular values. For simplicity we assume that $k=1$, the general case can be treated similarly. Since the off-diagonal blocks of $A_{i}$ have rank at most 1 then $C_{i}=u_{i} v_{i}^{T}, i=-1,0,1$, for suitable vectors $u_{i}, v_{i}$ where we assume that $\left\|u_{i}\right\|_{2}=\left\|C_{i}\right\|_{2},\left\|v_{i}\right\|_{2}=1$. Thus, we have $C(z)=\sum_{i=-1}^{1} z^{i} u_{i} v_{i}^{T}$. Since $I-D(z)$ is invertible on $\mathbb{A}$, we have $\tilde{C}(z)=H(z) \sum_{i=-1}^{1} z^{i} u_{i} v_{i}^{T} K(z)$ where $H(z)=(I-D(z))^{-1}, K(z)=S_{D}(z)^{-1}=\tilde{E}(z)$, and $H(z), K(z)$ are analytic for $z \in \mathbb{A}$. Consider the Fourier series of $H(z)$ and $K(z)$, that is, $H(z)=\sum_{s \in \mathbb{Z}} z^{s} H_{s}, K(z)=\sum_{s \in \mathbb{Z}} z^{s} K_{s}$, and recall that the coefficients $H_{s}, K_{s}$ have an exponential decay [13, Theorem 4.4c], that is, $\left|\left(H_{s}\right)_{i, j}\right| \leqslant \max _{z \in \mathbb{A}}\left|(H(z))_{i, j}\right| e^{-|s| \log t},\left|\left(K_{s}\right)_{i, j}\right| \leqslant$ $\max _{z \in \mathbb{A}}\left|(K(z))_{i, j}\right| e^{-|s| \log t}$. Since for any matrix norm induced by an absolute norm $\|\cdot\|$ and for any matrix $A$ it holds that $\left|a_{i, j}\right| \leqslant\|A\|$ so that we may write

$$
\left\|H_{s}\right\| \leqslant \max _{z \in \mathbb{A}}\|H(z)\| e^{-|s| \log t}, \quad\left\|K_{s}\right\| \leqslant \max _{z \in \mathbb{A}}\|K(z)\| e^{-|s| \log t} .
$$

Now recall that $\tilde{C}=H(z) \sum_{i=-1,0,1} z^{i} u_{i} v_{i}^{T} K(z)$, set $z \in \mathbb{T}$ and consider the generic ith term $z^{i} H(z) u_{i} v_{i}^{T} K(z)$ in the above summation. We have

$$
z^{i} H(z) u_{i} v_{i}^{T} K(z)=\sum_{s, h \in \mathbb{Z}} z^{s+h+i} H_{s} u_{i} v_{i} K_{h}=\sum_{s \in \mathbb{Z}} H_{s} u_{i} \sum_{p \in \mathbb{Z}} z^{p+i} v_{i}^{T} K_{p-s},
$$

where we have set $p=s+h$. Now, applying the operator $I_{N}$ to the above matrix cancels out the terms in $z^{p+i}$ such that $p+i$ is not multiple of $N$, so that we are left with the terms where $p+i=N q$ and we get

$$
I_{N}\left(z^{i} H(z) u_{i} v_{i}^{T} K(z)\right)=\sum_{s \in \mathbb{Z}} H_{s} u_{i} \sum_{q \in \mathbb{Z}} z^{q} v_{i}^{T} K_{N q-i-s}=: \sum_{s \in \mathbb{Z}} \hat{u}_{s}^{(i)} \hat{v}_{s}^{(i)}(z),
$$

for $\hat{u}_{s}^{(i)}=H_{s} u_{i}, \hat{v}_{s}^{(i)}(z)=\sum_{q \in \mathbb{Z}} z^{q} v_{i}^{T} K_{N q-i-s}$. Thus we may write

$$
I_{N}(\tilde{C})=\sum_{s \in \mathbb{Z}} \hat{U}_{s} \hat{V}_{s}(z)^{T}, \quad \hat{U}_{s}=\left[\hat{u}_{s}^{(-1)}, \hat{u}_{s}^{(0)}, \hat{u}_{s}^{(1)}\right], \quad \hat{V}_{s}(z)=\left[\hat{v}_{s}^{(-1)}(z), \hat{v}_{s}^{(0)}(z), \hat{v}_{s}^{(1)}(z)\right] .
$$

To complete the proof, recall that $z \in \mathbb{T}$ and apply Lemma 3.4 with $k=3$ to the series $\sum_{s \in \mathbb{Z}} \hat{U}_{s} V_{s}^{T}$. In order to do this, we have to provide upper bounds to $\left\|\hat{U}_{s} \hat{V}_{s}(z)^{T}\right\|_{2}$ for $z \in \mathbb{T}$. We have $\left\|\hat{U}_{s} \hat{V}_{s}(z)^{T}\right\|_{2} \leqslant\left\|\hat{U}_{s}\right\|_{2}\left\|\hat{V}_{s}(z)\right\|_{2}$. Concerning $\left\|\hat{U}_{s}\right\|_{2}$, since $\hat{U}_{s}=H_{s}\left[u_{-1}, u_{0}, u_{1}\right]$, we have $\left\|\hat{U}_{s}\right\|_{2} \leqslant\left\|H_{s}\right\|_{2}\left\|\left[u_{-1}, u_{0}, u_{1}\right]\right\|_{2} \leqslant \sqrt{3}\left\|H_{s}\right\|_{2} \max _{i}\left\|C_{i}\right\|_{2}$, where the latter inequality follows from the fact that $\left\|u_{i}\right\|_{2}=\left\|C_{i}\right\|_{2}$ and that consequently, $\left\|\left[u_{-1}, u_{0}, u_{1}\right]\right\|_{2} \leqslant \sqrt{3} \max _{i}\left\|C_{i}\right\|_{2}$. Thus from (6) we get

$$
\left\|\hat{U}_{s}\right\|_{2} \leqslant \sqrt{3} L \max _{z \in \mathbb{A}}\left\|H(z)^{-1}\right\|_{2} e^{-|s| \log t} .
$$

Similarly, since $\left\|v_{i}\right\|_{2}=1$ and $|z|=1$, we have

$$
\left\|\hat{v}_{s}^{(i)}\right\|_{2} \leqslant \sum_{q \in \boldsymbol{Z}}\left\|K_{N q-i-s}\right\|_{2} \leqslant \max _{z \in \mathbb{A}}\|K(z)\|_{2} \sum_{q \in \boldsymbol{Z}} e^{-|N q-i-s| \log t},
$$

where the last inequality follows from (6). Define $r$ the remainder of the division of $i+s$ by $N$, so that $i+s=N \hat{q}+r$, and get $\sum_{q \in Z} e^{-|N q-i-s| \log t}=\sum_{q \in Z} e^{-|N(q-\hat{q})+r| \log t}=\sum_{q \in Z} e^{-|N q+r| \log t}=e^{-r \log t}+\sum_{q \geqslant 1} e^{-(N q-r) \log t}+\sum_{q \geqslant 1} e^{-(N q+r) \log t}=$ $e^{-r \log t}+\left(e^{r \log t}+e^{-r \log t}\right)\left(\frac{1}{1-e^{-N \log t}}-1\right) \leqslant \frac{2}{1-e^{-N \log t}}$. Whence we deduce that 


$$
\left\|\hat{V}_{s}\right\|_{2} \leqslant \frac{2 \sqrt{3}}{1-e^{-N \log t}} \max _{z \in \mathbb{A}}\|K(z)\|_{2} .
$$

Combining the two bounds yields

$$
\left\|\hat{U}_{S} \hat{V}_{s}(z)\right\|_{2} \leqslant \frac{6 L}{1-e^{-N \log t}} \max _{z \in \mathbb{A}}\|K(z)\|_{2} \max _{z \in \mathbb{A}}\|H(z)\|_{2} e^{-|s| \log t} .
$$

It remains to estimate $\|K(z)\|_{2}$ and $\|H(z)\|_{2}$. Concerning $K(z)=\tilde{E}(z)$, observe that this is a principal submatrix of $\psi(z)$ so that $\|K(z)\|_{2} \leqslant\|\psi(z)\|_{2}$. Concerning $H(z)=(I-D(z))^{-1}$, observe that from the condition $A_{i} \geqslant 0$ it follows that $|D(z)| \leqslant$ $D(|z|)$ and that $\rho(D(z)) \leqslant \rho(|D(z)|) \leqslant \rho(D(|z|)) \leqslant \rho(\varphi(|z|))<1$ since $I-D(z)$ is a principal submatrix of $\varphi(z)$. Thus we may write $(I-D(z))^{-1}=\sum_{j=0}^{\infty} D(z)^{j}$ and $\left|(I-D(z))^{-1}\right| \leqslant(I-D(|z|))^{-1}$. Now, since $A_{i} \geqslant 0$ for $i=-1,0,1$, then

$$
\tilde{D}(|z|)=(I-D(|z|))^{-1}+\underbrace{(I-D(|z|))^{-1}}_{\geqslant 0} \underbrace{C(|z|)}_{\leqslant 0} \underbrace{S_{I-D(|z|)}^{-1}}_{\geqslant 0} \underbrace{B(|z|)}_{\leqslant 0} \underbrace{(I-D(|z|))^{-1}}_{\geqslant 0} \geqslant(I-D(|z|))^{-1}
$$

so that $\left\|(I-D(z))^{-1}\right\|_{2} \leqslant\left\|(I-D(|z|))^{-1}\right\|_{2} \leqslant\|\tilde{D}(|z|)\|_{2} \leqslant \max _{z \in \mathbb{A}}\|\psi(z)\|_{2}$. Thus, applying Lemma 3.4 together with the bound (7) and rank of the blocks 3 yields

$$
\sigma_{s}\left(\tilde{C}^{(h)}(z)\right) \leqslant \frac{12 L \delta^{2}}{\left(1-e^{-N \log t}\right)\left(1-t^{-1}\right)} e^{-\frac{s-3}{6} \log t} .
$$

If the blocks $A_{i}$ are $k$-quasiseparable, then Lemma 3.4 is applied with rank of the blocks $3 k$ so that the exponent $(s-3) / 6$ is replaced by $(s-3 k) /(6 k)$. If $\varphi(z)$ is tridiagonal, then $u_{-1}=u_{0}=u_{1}$ and $v_{-1}=v_{0}=v_{1}$, so that $\hat{U}_{j}$ and $\hat{V}_{j}$ are formed by a single column, i.e., Lemma 3.4 is applied with rank of the blocks 1 . This provides (5).

\subsection{Exponential decay of the singular values in $\varphi(z)$}

In this section, we prove the decay property of the singular values in the off-diagonal submatrices of $\varphi^{(h)}(z)$ when $|z|=1$. The proof is obtained by combining the decay property for the matrix function $\psi^{(h)}$, stated in Theorem 4.1 , with a suitable lemma which allows to extend this property to the matrix inverse.

Lemma 4.2. Let $\varphi^{(h)}(z)=-z^{-1} A_{-1}^{(h)}+I-A_{0}^{(h)}-z A_{1}^{(h)}$ be the $m \times m$-matrix Laurent polynomial obtained at the hth step of $C R$. Under the hypotheses of Theorem 4.1, for every $z \in \mathbb{T}$ we have the following bound:

$$
\sigma_{j}\left(C^{(h)}\right) \leqslant K\left(L_{h}, \varphi\right) \cdot \sigma_{j}\left(\tilde{C}^{(h)}\right), \quad K\left(L_{h}, \varphi\right)=\left(1+3 L_{h}\right)\left(1+L_{h}+L_{h}^{2}\left\|\varphi(1)^{-1}\right\|_{2}\right)
$$

where $\varphi^{(h)}(z)$ and $\varphi^{(h)}(z)^{-1}$ are partitioned as in (3) and $L_{h}$ is such that $\left\|A_{i}^{(h)}\right\|_{2} \leqslant L_{h}$.

Proof. With the notation of the partitioning (3) applied to $\varphi^{(h)}(z)$, from Lemma 3.3 applied to $\varphi^{(h)}(z)$ we have $\sigma_{j}\left(C^{(h)}\right) \leqslant\left\|I-E^{(h)}(z)\right\|_{2}\left\|S_{I-E^{(h)}}(z)\right\|_{2} \sigma_{j}\left(\tilde{C}^{(h)}\right)$. Thus, since $z \in \mathbb{T}$ and $I-E^{(h)}(z)$ is a submatrix of $\varphi^{(h)}(z)$, we have $\left\|I-E^{(h)}(z)\right\|_{2} \leqslant\left\|\varphi^{(h)}(z)\right\|_{2} \leqslant 1+3 L_{h}$. Moreover, taking the norms in $S_{I-E^{(h)}}(z)=I-D^{(h)}(z)-C^{(h)}(z)\left(I-E^{(h)}(z)\right)^{-1} B^{(h)}(z)$ we get $\left\|S_{I-E^{(h)}}(z)\right\|_{2} \leqslant 1+L_{h}+L_{h}\left\|\left(I-E^{(h)}(z)\right)^{-1}\right\|_{2} L_{h}$. Moreover, for $z \in \mathbb{T}$ we have $\left.\mid I-E^{(h)}(z)\right)^{-1} \mid \leqslant \sum_{i=0}^{\infty} E^{(h)}(1)^{i}$ so that $\left.\| I-E^{(h)}(z)\right)^{-1}\left\|_{2} \leqslant\right\|\left(I-E^{(h)}(1)\right)^{-1}\left\|_{2}=\right\| \sum_{i=0}^{\infty} E^{(h)}(1)^{i}\left\|_{2} \leqslant\right\| \sum_{i=0}^{\infty} A^{(h)}(1)^{i}\left\|_{2}=\right\| \varphi^{(h)}(1)^{-1} \|_{2}$, where we have set $A^{(h)}(z)=$ $z^{-1} A_{-1}^{(h)}+A_{0}^{(h)}+z A_{1}^{(h)}$. Here, we have used the property that the conditions $A_{-1}^{(h)}, A_{0}^{(h)}, A_{1}^{(h)} \geqslant 0$ and $\rho\left(A_{-1}^{(h)}+A_{0}^{(h)}+A_{1}^{(h)}\right)<1$ are preserved at each step of CR (see [2]). Finally, since $\varphi^{(h)}(1)^{-1}=\psi^{(h)}(1)=\frac{1}{N} \sum_{i=0}^{N-1} \psi\left(\omega_{N}^{i}\right)$, for $N=2^{h}$ (see Section 2), we have $\left\|\varphi^{(h)}(1)^{-1}\right\|_{2} \leqslant\|\psi(1)\|_{2}$.

Remark 4.3. Note that the previous bound still holds with $\left\|\varphi^{(h)}(1)^{-1}\right\|_{2}$, in place of $\left\|\varphi(1)^{-1}\right\|_{2}$. Experimentally, $\left\|\varphi^{(h)}(1)^{-1}\right\|_{2}$ is much smaller than $\left\|\varphi(1)^{-1}\right\|_{2}$ just after few steps $h$.

Observe that $L_{h}$ depends on the step $h$ of CR. However, since under the assumptions of Theorem 4.1, the sequences generated by CR are such that $\lim _{k} A_{i}^{(h)}=0$, for $i=1,-1$ while $\lim _{h} A_{0}^{(h)}$ is finite (see [2]), then there exists $L$ such that $L \geqslant L_{h}$. Thus, combining Lemma 4.2 and Theorem 4.1 we obtain the following result.

Corollary 4.4. Let $\varphi^{(h)}(z)=-z^{-1} A_{-1}^{(h)}+I-A_{0}^{(h)}-z A_{1}^{(h)}$ be the $m \times m$-matrix Laurent polynomial obtained at the hth step of CR and assume the hypothesis of Theorem 4.1. Then for any off-diagonal submatrix $C^{(h)}(z)$ of $\varphi^{(h)}(z)$ we have $\sigma_{s}\left(C^{(h)}\right) \leqslant 3 M K \cdot e^{\frac{s-3 k}{6 k} \log t}$, where $K=(1+3 L)\left(1+L+L^{2}\left\|\varphi(1)^{-1}\right\|_{2}\right), M$ is the constant defined in Theorem 4.1 and $L \geqslant\left\|A_{i}^{(h)}\right\|_{2}$, for $i=-1,0$, 1. In particular, if $A_{i}$ is tridiagonal for $i=-1,0,1$ then $\sigma_{s}\left(C^{(h)}\right) \leqslant M K \cdot e^{-\left(\frac{s}{2}\right) \log t}$ 


\subsection{Exponential decay of the singular values in $A_{i}^{(h)}$}

To prove the decay of the singular values in the off-diagonal submatrices of $A_{i}^{(h)}$ for $i=-1,0,1$ we rely on the following result of which we omit the elementary proof.

Lemma 4.5. Let $A(z)=z^{-1} A_{-1}+A_{0}+z A_{1}$ and let $\xi$ be a primitive 6-th root of the unity. Then $A_{-1}=\frac{1}{3}\left(\xi A(\xi)+\xi^{5} A\left(\xi^{5}\right)-A(-1)\right)$, $A_{0}=\frac{1}{2}(A(z)+A(-z)), A_{1}=\frac{1}{3}\left(\xi^{5} A(\xi)+\xi A\left(\xi^{5}\right)-A(-1)\right)$.

Lemma 4.6. Let $A=\frac{1}{k} \sum_{i=1}^{k} A_{i} \in \mathbb{C}^{n \times n}$ where $\sigma_{j}\left(A_{i}\right) \leqslant \gamma e^{-\alpha j}$, for $j=1, \ldots, n$. Then $\sigma_{j}(A) \leqslant \tilde{\gamma} e^{-\alpha \frac{j-k}{k}}, \tilde{\gamma}=\frac{\gamma}{1-e^{-\alpha}}$.

Proof. Relying on the SVD, we write $A_{i}=\sum_{j=1}^{\infty} \sigma_{j}\left(A_{i}\right) u_{i, j} v_{i, j}^{*}$ where $u_{i, j}$ and $v_{i, j}$ are the singular vectors of $A_{i}$ and where, for convenience, we have expanded the sum to an infinite number of terms by $\operatorname{setting} \sigma_{j}\left(A_{i}\right)=0$ for $j>n$. This allows us to write

$$
A=\frac{1}{k} \sum_{i=1}^{k} A_{i}=\sum_{j=1}^{\infty}\left(\frac{1}{k} \sum_{i=1}^{k} \sigma_{j}\left(A_{i}\right) u_{i, j} v_{i, j}^{*}\right)=\sum_{j=1}^{\infty} \tilde{A}_{j}
$$

Observe that $\tilde{A}_{j}$ have rank $k$ and $\left\|A_{j}\right\| \leqslant \gamma e^{-\alpha j}$. Applying Lemma 3.4 completes the proof.

We may conclude with the decay property for the singular values of the off-diagonal submatrices of $A_{i}^{(h)}$, for $i=-1,0,1$.

Lemma 4.7. Let $\varphi^{(h)}(z)$ be the matrix function generated at the hth step of CR with the property that every offdiagonal submatrix $B(z)$ of $\varphi^{(h)}(z)$ has decaying singular values such that $\sigma_{s}(B(z)) \leqslant \gamma e^{-\alpha s}$. Then every coefficient $B_{i}$ of $B(z)=z^{-1} B_{-1}+B_{0}+z B_{1}$ is such that $\sigma_{s}\left(B_{0}\right) \leqslant \gamma e^{-\alpha \frac{j-2}{2}}, \sigma_{s}\left(B_{i}\right) \leqslant \gamma e^{-\alpha \frac{j-3}{3}}$, for $i=1,-1$.

Proof. By Lemma 4.5, we have an expression for $B_{i}$ based on evaluations of $B(z)$. In particular, we have $A_{0}=\frac{1}{2}(B(i)+$ $B(-i)), A_{ \pm 1}=\frac{1}{3}\left(\xi^{\mp 1} B(\xi)+\xi^{\mp 5} B\left(\xi^{5}\right)-B(-1)\right)$, where $\xi$ is a primitive 6-th root of the unity. Applying Lemma 4.6 completes the proof.

\subsection{The Markovian case}

One of the most interesting application of CR algorithm is in the Markovian framework, in which applicability and convergence properties are guaranteed. In that case, the matrix function $\varphi$ satisfies almost all the hypotheses made in the previous subsections but it is singular at $z=1$ since 1 is always an eigenvalue of $\varphi(z)$. Nevertheless we will show that Corollary 4.4 can still be applied considering a rescaled version of $\varphi(z)$.

When the coefficients $A_{i}$ for $i=-1,0,1$ represent the blocks of the transition matrix of an irreducible not null recurrent QBD process, the eigenvalues of $\varphi(z)$ enjoy the following properties [10,4]:

(i) $\left|\lambda_{1}\right| \leqslant\left|\lambda_{2}\right| \leqslant \cdots \leqslant\left|\lambda_{m-1}\right| \leqslant \lambda_{m}<\lambda_{m+1} \leqslant\left|\lambda_{m+2}\right| \leqslant \cdots \leqslant\left|\lambda_{2 m}\right|$, with $\lambda_{m}, \lambda_{m+1} \in \mathbb{R}$ and one of the two equal to 1 .

(ii) In the annulus $\left\{\lambda_{m}<|z|<\lambda_{m+1}\right\} \varphi$ is invertible and the spectral radius of $I-\varphi(z)$ is strictly less than 1 .

Hence we consider the rescaled version of $\varphi$, that is, $\varphi_{\theta}(z):=\varphi(\theta z)$, and we choose $\theta=\sqrt{\lambda_{m} \lambda_{m+1}}$. We obtain a matrix function invertible on $\mathbb{A}=\left\{\frac{1}{t}<|z|<t\right\}$ where $t=\sqrt{\frac{\lambda_{m+1}}{\lambda_{m}}}$.

Observe that $\varphi_{\alpha}^{(h)}(z):=\varphi^{(h)}\left(\alpha^{2^{h}} z\right)$ so applying CR to $\varphi_{\alpha}$ one obtains the same matrix sequences up to a rescaling factor. In particular the exponential decay of the singular values is left unchanged as shown in the following.

Theorem 4.8. For given $t>1$ and $\delta \geqslant 0$, consider the following class of matrix functions associated with $Q B D$ stochastic processes with $k$-quasiseparable blocks:

$$
\chi_{\delta, t}:=\left\{\varphi(z):\left\|\varphi^{-1}(z)\right\|_{2} \leqslant \delta \quad t^{-1} \leqslant|z| \leqslant t, \quad t<\lambda_{m+1} / \lambda_{m}\right\} .
$$

Then there exists a uniform constant $\gamma(\delta, t)$ such that for any off-diagonal block $C^{(h)}(z)$ of $\varphi^{(h)}(z)$, with $\varphi \in \chi_{\delta, t}$, its s-th singular value is bounded by $\sigma_{s}\left(C^{(h)}(z)\right) \leqslant \gamma(\delta, t) \cdot e^{-\frac{s-3 k}{6 k} \log t}$.

Remark 4.9. Observe that in the case of null-recurrent QBD processes one has $\lambda_{m}=\lambda_{m+1}=1$, so that there is no open annulus including $\mathbb{T}$ where $\varphi(z)$ is nonsingular and we cannot apply Theorem 4.1. This drawback can be partially overcome 

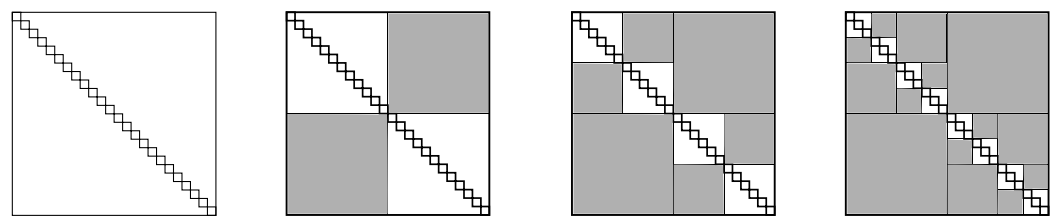

Fig. 2. The behavior of the block partitioning in the $\mathcal{H}$-matrix representation. The blocks filled with grey are represented as sum of dyads, the diagonal blocks in the last step are stored as full matrices.

by applying the shift technique of $[2,4]$. This technique allows to construct a new matrix function $\tilde{\varphi}(z)$ which has the same eigenvalues of $\varphi(z)$ except for the eigenvalue 1 which is shifted to 0 . So that $\tilde{\varphi}(z)$ has an open annulus containing $\mathbb{T}$ where it is nonsingular. Moreover, applying $\mathrm{CR}$ to $\tilde{\varphi}(z)$ generates matrix sequences which easily allow to recover the corresponding matrix sequences obtained by applying $\mathrm{CR}$ to $\varphi(z)$. The sequences associated with $\varphi(z)$ differ from the sequences associated with $\tilde{\varphi}(z)$ by a rank-1 correction. This way, if the exponential decay of the singular values holds for the latter sequences, it holds also for the former ones. The difficulty that still remains is that the nonnegativity of the blocks $A_{-1}, A_{0}$ and $A_{1}$ is not generally satisfied by the function $\tilde{\varphi}(z)$ so that in principle Theorem 4.1 cannot be applied and a different version specific for this case should be formulated.

Remark 4.10. The bounds that we have given to the decay of the singular values of the off-diagonal submatrices are not strict. Experimentally, singular values seem to decay slightly faster. Even in the null-recurrent case where $\lambda_{m}=\lambda_{m+1}=1$, the decay still occurs even though in a deteriorated form. The decay properties clearly depend on the domain of analyticity of $\psi(z)$ but this is not the only reason of the decay. More investigation is needed in this direction.

\section{An algorithm using $\mathcal{H}$-matrices}

We have provided an implementation of $\mathrm{CR}$, which applies to matrix functions $\varphi(z)$ having quasiseparable blocks, and relies on the approximate quasiseparable structure induced by the decay of the singular values. We relied on the $\mathcal{H}$-matrix representation of $[6,7,11]$.

\section{1. $\mathcal{H}$-matrix representation}

Here, we give a brief and informal description of the $\mathcal{H}$-matrix representation that we have implemented. For full details we refer to [6] where an overview of the definition and use of hierarchical matrices is given.

Let $A \in \mathbb{R}^{n \times n}$ be a $k$-quasiseparable matrix such that $A=\left[\begin{array}{l}A_{11} A_{22} \\ A_{21} A_{22}\end{array}\right], A_{11} \in \mathbb{R}^{n_{1} \times n_{1}}, A_{22} \in \mathbb{R}^{n_{2} \times n_{2}}$, with $n_{1}:=\left\lfloor\frac{n}{2}\right\rfloor$ and $n_{2}:=\left\lceil\frac{n}{2}\right\rceil$. Observe that the antidiagonal blocks $A_{12}$ and $A_{21}$ do not involve any element of the main diagonal of $A$, hence they are representable as a sum of at most $k$ dyads. Moreover the diagonal blocks $A_{11}$ and $A_{22}$ are square matrices with the same rank structure of $A$. Therefore these diagonal blocks are recursively represented with a similar partitioning. If blocks become small enough, they are stored as full matrices (Fig. 2).

\subsection{Quasiseparable CR}

If the quasiseparable rank of the $\mathcal{H}$-matrices we are dealing with can be treated as a constant when compared to the dimension $n$, then the algorithms which perform the arithmetic operations have almost linear complexity [6, Chapter 6]. In particular we can achieve complexity $O(n \log n)$ for matrix addition and $O\left(n \log ^{2} n\right)$ for matrix multiplication and inversion. This is almost optimal, provided that the rank remains sufficiently low.

In order to fully exploit the numerical quasiseparable structure we perform the arithmetic operations of CR adaptively with respect to the rank of the blocks. This means that the result of an arithmetic operation (e.g. matrix multiplication) will be an $\mathcal{H}$-matrix with the same partitioning, where each low rank block is a truncated reduced SVD of the corresponding block of the exact result. Hence the rank is not a priori fixed but depends on a threshold $\epsilon$ at which the truncation is done. The parameter $\epsilon$ can be regarded as the desired accuracy (for us is close to the machine precision $2.22 \times 10^{-16}$ ) and can be crucial for the performance of the algorithm.

\subsection{A note on the implementation}

For the implementation of this algorithm we relied on the open source library H2Lib [5]. The library has been wrapped in MEX files for use in MATLAB, where the numerical experiments have been run. The code developed in this context is freely available at [16]. The bindings developed in the testing of the algorithm are only a partial mapping of all the routines available in the original H2Lib library but we feel that it is worth making them public so they can be used as a base for a further extension. 
Table 1

Timings and accuracy for 15 iterations of $C R$ at the increasing of the size of the blocks.

\begin{tabular}{|c|c|c|c|c|c|c|c|c|}
\hline & CR & & $H_{10^{-16}}$ & & $H_{10^{-12}}$ & & $H_{10^{-8}}$ & \\
\hline Size & Time $(\mathrm{s})$ & Residue & Time (s) & Residue & Time (s) & Residue & Time $(\mathrm{s})$ & Residue \\
\hline 100 & $6.04 e-02$ & $1.91 e-16$ & $2.21 e-01$ & $1.79 e-15$ & $2.04 e-01$ & $8.26 e-14$ & $1.92 e-01$ & $7.40 e-10$ \\
\hline 200 & $1.88 e-01$ & $2.51 e-16$ & $5.78 e-01$ & $1.39 e-14$ & $5.03 e-01$ & $1.01 e-13$ & $4.29 e-01$ & $2.29 e-09$ \\
\hline 400 & $1.61 e+01$ & $2.09 e-16$ & $3.32 e+00$ & $1.41 e-14$ & $2.60 e+00$ & $1.33 e-13$ & $1.98 e+00$ & $1.99 e-09$ \\
\hline 800 & $2.63 e+01$ & $2.74 e-16$ & $4.55 e+00$ & $1.94 e-14$ & $3.49 e+00$ & $2.71 e-13$ & $2.63 e+00$ & $2.69 e-09$ \\
\hline 1600 & $8.12 e+01$ & $3.82 e-12$ & $1.18 e+01$ & $3.82 e-12$ & $8.78 e+00$ & $3.82 e-12$ & $6.24 e+00$ & $3.39 e-09$ \\
\hline 3200 & $6.35 e+02$ & $5.46 e-08$ & $3.12 e+01$ & $5.46 e-08$ & $2.21 e+01$ & $5.46 e-08$ & $1.51 e+01$ & $5.43 e-08$ \\
\hline 6400 & $5.03 e+03$ & $3.89 e-08$ & $7.83 e+01$ & $3.89 e-08$ & $5.38 e+01$ & $3.89 e-08$ & $3.58 e+01$ & $3.87 e-08$ \\
\hline 12800 & $4.06 e+04$ & $1.99 e-08$ & $1.94 e+02$ & $1.99 e-08$ & $1.29 e+02$ & $1.99 e-08$ & $8.37 e+01$ & $1.97 e-08$ \\
\hline
\end{tabular}

Table 2

Timings and accuracy for 15 iterations of CR on blocks with size 1600 with different bands.

\begin{tabular}{|c|c|c|c|c|c|c|c|c|}
\hline & CR & & $H_{10^{-16}}$ & & $H_{10^{-12}}$ & & $H_{10^{-8}}$ & \\
\hline Band & Time $(\mathrm{s})$ & Residue & Time (s) & Residue & Time (s) & Residue & Time $(\mathrm{s})$ & Residue \\
\hline 2 & $7.47 e+01$ & $2.11 e-16$ & $1.58 e+01$ & $6.95 e-15$ & $1.08 e+01$ & $2.62 e-13$ & $7.86 e+00$ & $2.57 e-09$ \\
\hline 4 & $7.65 e+01$ & $1.66 e-16$ & $1.92 e+01$ & $4.88 e-15$ & $1.48 e+01$ & $2.36 e-13$ & $9.44 e+00$ & $3.15 e-09$ \\
\hline 8 & $7.82 e+01$ & $1.48 e-16$ & $2.81 e+01$ & $6.11 e-15$ & $2.15 e+01$ & $2.08 e-13$ & $1.31 e+01$ & $2.10 e-09$ \\
\hline 16 & $7.50 e+01$ & $1.35 e-16$ & $4.99 e+01$ & $4.98 e-15$ & $3.48 e+01$ & $2.29 e-13$ & $2.28 e+01$ & $2.08 e-09$ \\
\hline 32 & $7.97 e+01$ & $1.33 e-16$ & $9.40 e+01$ & $5.79 e-15$ & $6.32 e+01$ & $2.01 e-13$ & $4.15 e+01$ & $2.28 e-09$ \\
\hline 64 & $8.03 e+01$ & $1.31 e-16$ & $1.97 e+02$ & $6.79 e-15$ & $1.29 e+02$ & $1.99 e-13$ & $8.37 e+01$ & $2.01 e-09$ \\
\hline 128 & $7.53 e+01$ & $1.28 e-16$ & $4.01 e+02$ & $5.89 e-15$ & $2.71 e+02$ & $2.02 e-13$ & $1.75 e+02$ & $2.15 e-09$ \\
\hline
\end{tabular}
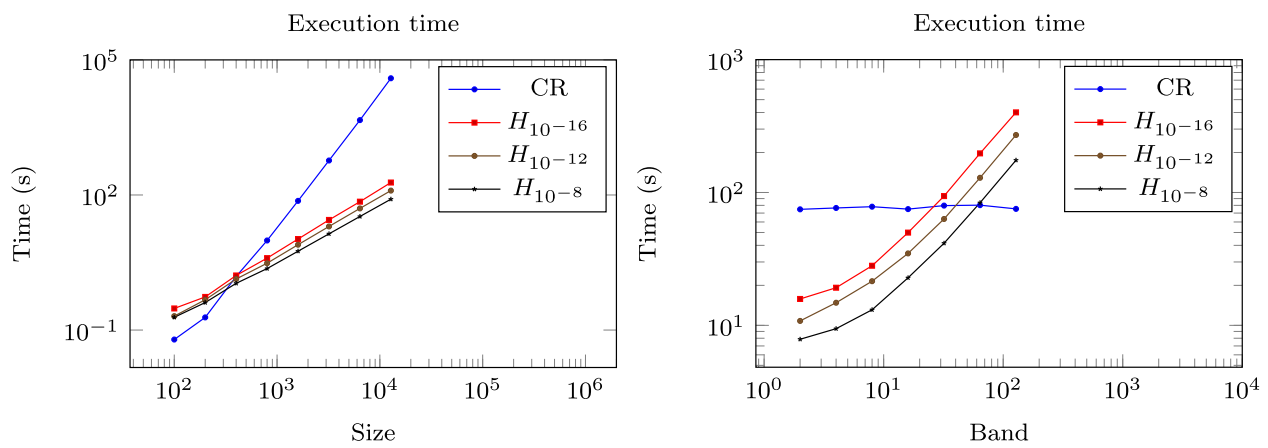

Fig. 3. Timings of CR. To the left, CR is applied to tridiagonal blocks with increasing size. To right, CR is applied to band blocks with increasing band and size 1600. (For interpretation of the references to color in this figure legend, the reader is referred to the web version of this article.)

For a fair comparison, we have compiled H2Lib with the LAPACK library used by MATLAB. Moreover, we have disabled the parallelism in the Intel MKL library to obtain more accurate results. It is important to notice that running with parallelism enabled in the MKL library leads to improved performance both for H2LIb and for MATLAB, but the improvement is more relevant in the latter. This is due to the fact that the library is optimized for the multiplication of large matrices, such as in the full CR implementation (when full matrices of large size are multiplied together). The multiplication of the small rectangular matrices involved in the hierarchical representation, instead, benefit less from this implementation. Anyway, also in this case we see that our implementation is more efficient even if starting from larger dimension. For example, on a Xeon server with 24 threads available our implementation is faster than the standard one approximately for $n>500$.

Table 1 reports the results of some numerical experiments, where in each column we have reported: the size of the blocks from $m=100$ up to $m=12800$, the CPU time, in seconds, required by standard CR and the residual error, then from column 3 to column 5 we reported the CPU time, in seconds, and the residual error of our implementation with values of $\epsilon=10^{-16}, 10^{-12}, 10^{-8}$, respectively. It is interesting to observe that the precision of the result does not deteriorate much for large values of $m$. Moreover, the speed-up that we get goes beyond two order of magnitude.

In Table 2 we repeat the experiment fixing the size to 1600 and letting the band of the starting blocks to increase exponentially from 2 up to 128 . It should be note that the gain of time of our implementation seems to deteriorate linearly with respect to the increase of the band.

In Fig. 3 we give a graphic description, in logarithmic scale, of the growth of the CPU time in the latter experiments. The test problems are generated randomly. 


\section{Conclusions}

We have experimentally observed the exponential decay of the singular values of certain off-diagonal submatrices generated by cyclic reduction applied to certain QBD stochastic processes of practical interest. We have formally related this property to the width of the domain of analyticity of the inverse matrix function associated with the QBD.

We have provided a software implementation of $\mathrm{CR}$, for $\mathrm{QBD}$ with tridiagonal blocks encountered in the analysis of bidimensional random walk, which relies on this decay property. The speed up that we get with respect to standard CR is substantial even with moderately large size of the blocks.

Even though experiments confirm the validity of the theoretical bounds, the bounds obtained in our analysis are not sharp with respect to the values actually encountered in our computational experiments. This shows that the decay property depends also on other factors which deserve further investigation. This is the aim of our future research.

\section{References}

[1] D.A. Bini, P. Favati, B. Meini, A compressed cyclic reduction for QBD processes with low-rank upper and lower transitions, in: Matrix-Analytic Methods in Stochastic Models, Springer, 2013, pp. 25-40

[2] D.A. Bini, G. Latouche, B. Meini, Numerical Methods for Structured Markov Chains, Oxford University Press, 2005.

[3] D.A. Bini, B. Meini, Effective methods for solving banded Toeplitz systems, SIAM J. Matrix Anal. Appl. 20 (1999) 700-719.

[4] D.A. Bini, B. Meini, The cyclic reduction algorithm: from Poisson equation to stochastic processes and beyond, Numer. Algorithms 51 (2009) $23-60$.

[5] S. Börm, H2Lib, Available from GitHub at https://github.com/H2Lib/H2Lib, 2015.

[6] S. Börm, L. Grasedyck, W. Hackbusch, Hierarchical Matrices, Lect. Notes, vol. 21, 2003, p. 2003.

[7] S. Börm, L. Grasedyck, W. Hackbusch, Introduction to hierarchical matrices with applications, Eng. Anal. Bound. Elem. 27 (2003) $405-422$.

[8] B.L. Buzbee, G.H. Golub, C.W. Nielson, On direct methods for solving Poisson's equations, SIAM J. Numer. Anal. 7 (1970) 627-656.

[9] S. Chandrasekaran, P. Dewilde, M. Gu, N. Somasunderam, On the numerical rank of the off-diagonal blocks of Schur complements of discretized elliptic PDEs, SIAM J. Matrix Anal. Appl. 31 (2010) 2261-2290

[10] H. Gail, S. Hantler, B. Taylor, Matrix-geometric invariant measures for g/m/l type Markov chains, Commun. Stat., Stoch. Models 14 (1998) 537-569.

[11] L. Grasedyck, W. Hackbusch, Construction and arithmetics of h-matrices, Computing 70 (2003) 295-334.

[12] C.H. Guo, Convergence analysis of the Latouche-Ramaswami algorithm for null recurrent quasi-birth-death processes, SIAM J. Matrix Anal. Appl. 23 (2002) 744-760.

[13] P. Henrici, Applied and Computational Complex Analysis. Vol. 1, Wiley Classics Library, John Wiley \& Sons, Inc., New York, 1988. Power seriesintegration-conformal mapping-location of zeros, Reprint of the 1974 original, A Wiley-Interscience Publication.

[14] R.W. Hockney, A fast direct solution of Poisson's equation using Fourier analysis, J. ACM 12 (1965) 95-113.

[15] J.R. Jackson, Networks of waiting lines, Oper. Res. 5 (1957) 518-521.

[16] S. Massei, L. Robol, H2lib’s MATLAB bindings, Available from GitHub at https://github.com/robol/h2lib-matlab, 2015.

[17] M. Miyazawa, Tail decay rates in double QBD processes and related reflected random walks, Math. Oper. Res. 34 (2009) 547-575.

[18] J.F. Pérez, B. Van Houdt, Quasi-birth-and-death processes with restricted transitions and its applications, Perform. Eval. 68 (2011) $126-141$.

[19] R. Vandebril, M. Van Barel, N. Mastronardi, Matrix Computations and Semiseparable Matrices. Linear Systems, vol. 1, Johns Hopkins University Press, Baltimore, MD, 2008. 